\title{
Evaluación Estadística del Rendimiento Operativo en Limas de Producción Macional
}

\author{
Helmer Galvis* \\ John Jairo Coronado* \\ José Isidro García*
}

* MSc. Ingenieros Mecánicos - Profesores Escuela de Ingeniería Mecánica - Universidad del Valle - Santiago de Cali, Colombia

E-mail:
Fecha de recepción: Junio 13 de 2003

Fecha de aprobación: Marzo 26 de 2003

\section{RESUMEN}

En la industria manufacturera de herramientas la globalización exige cada día mayores estándares de calidad, lo que obliga a realizar modificaciones en el proceso de producción que necesitan ser cuantificadas a fin de evaluar los resultados generados. Este trabajo muestra un análisis de inferencia estadística para medir el rendimiento operativo entre tres limas de producción nacional de tres compañías diferentes. Utilizando un banco de pruebas que garantizó condiciones de operación similares (presión de operación constante y probeta estandarizada), se estimó el rendimiento operativo de las limas a partir del material removido en la probeta. Estos datos 
fueron analizados estadísticamente para validar las modificaciones realizadas en el proceso productivo. Finalmente, con los resultados obtenidos se podrán conocer los beneficios de las modificaciones de un proceso de producción.

\section{Palabras Claves:}

Inferencia estadística, lima, rendimiento operativo.

\section{ABSTRACT}

The globalization produces a higher quality standards in the tool manufacture industries, requiring to modify the manufacturing processes. They need to be quantified in order to evaluate the results. This paper shows a statistical analysis using inference techniques to measure the operative performance among three files of national manufacturers. In an experimental bank under similar operation conditions (constant pressure over the files and standarized samples) the files performance was calculated with the material removed on the sample. These data were statistically analyzed to validate the modifications carried out in the productive process. Finally, with this analysis will be possible to know if the modifications made on the production process are successful.

\section{Key Words:}

Statistical inference, file, operative performance.

\section{INTRODUCCIÓN}

La competencia entre las compañías lleva a un mejoramiento continuo de la calidad para ofrecer productos de mayores estándares obteniendo un dominio relativo en el mercado. Algunas modificaciones del proceso productivo pueden llevar a beneficios que deben ser analizados y cuantificados para evaluar su conveniencia. La teoría de inferencia estadística permite hacer generalizaciones acerca de una población a través de tamaño de muestras satisfactorio con los niveles de confianza deseados. La inferencia se divide en dos áreas principales como son la estimación y la prueba de hipótesis. El presente trabajo utilizó la estimación para evaluar el desempeño operativo de un tipo de lima fabricada por tres compañías nacionales. Esto se hizo midiendo el material removido en una probeta por las limas bajo condiciones de operación controladas. El propósito de este trabajo es comparar si las modificaciones del proceso productivo realizado por una de estas compañías fueron satisfactorias en la obtención de una lima de mejor desempeño que las fabricadas por la competencia.

\section{METODOLOGÍA}

Con el fin de evaluar los efectos de la modificación del proceso de fabricación de un tipo de lima de una compañía nacional, se comparó el rendimiento de este tipo de lima con las fabricadas por otras dos empresas. Se entiende por rendimiento el peso de material removido por una lima bajo condiciones estandarizadas. Para medir el rendimiento se utilizó un banco de pruebas (fig.1) controlando las variables de mayor relevancia como son la presión ejercida por la lima sobre la probeta, la longitud recorrida por la lima y los ciclos de operación.

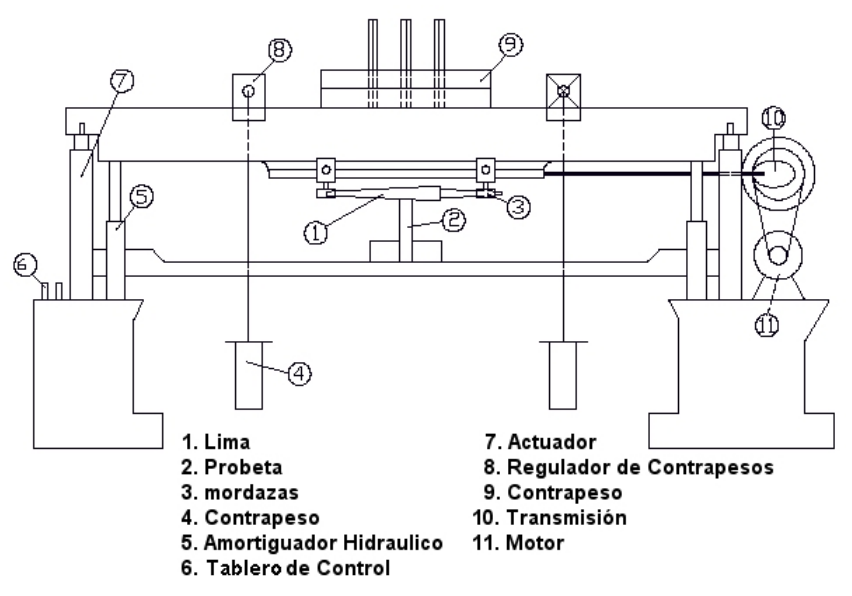

Figura 1. Banco de Pruebas

Se estimó el tamaño de muestra requerida para que la evaluación fuera estadísticamente significativo. Para el análisis de los datos se utilizó el método clásico de inferencia estadística a 
través de la estimación. Una vez definido el tamaño de muestra se realizó la compra de la cantidad de limas a diferentes proveedores en búsqueda de una muestra aleatoria, conformando tres espacios muestrales (limas del fabricante $A$, fabricante $B$ y fabricante C). Posteriormente, se midió el rendimiento de cada una de las limas de la muestra seleccionada, calculando la media y la desviación estándar del rendimiento de cada población. Con esta información se determinó un intervalo de confianza para los rendimientos de las limas de cada fabricante. Finalmente, se realizó una estimación de la diferencia de medias para identificar cual fabricante producía limas de mejor rendimiento.

\section{RESULTADOS Y CONCLUSIONES}

\section{Tamaño de la muestra}

Es indispensable realizar una selección adecuada del tamaño de la muestra para tener un nivel de incertidumbre satisfactorio de los resultados. El tamaño de la muestra para las limas se estimó con mediciones previas de las desviaciones estándar de rendimientos de limas de los fabricantes A, B y C. La Tabla 1 describe la cantidad de limas a ser analizadas para obtener resultados con cierto grado de confiabilidad.

\begin{tabular}{|c|c|c|c|c|c|c|}
\hline $\begin{array}{c}\text { Error Tipo I } \\
(\%)\end{array}$ & $\begin{array}{c}\text { Error Tipo II } \\
(\%)\end{array}$ & $\begin{array}{l}\text { Diferencia } \\
\text { (Gramos) }\end{array}$ & $\begin{array}{c}\text { Desviación } \\
\text { Estándar } \\
\text { Fabricante A }\end{array}$ & $\begin{array}{c}\text { Desviación } \\
\text { Estándar } \\
\text { Fabricante B }\end{array}$ & $\begin{array}{c}\text { Desviación } \\
\text { Estándar } \\
\text { Fabricante C }\end{array}$ & $\begin{array}{c}\text { Tamaño de } \\
\text { Muestra }\end{array}$ \\
\hline 0.05 & 0.05 & 3 & 0.7 & 1.4 & - & 4 \\
\hline 0.05 & 0.05 & 4 & 0.7 & - & 0.8 & 1 \\
\hline 0.01 & 0.01 & 3 & 0.7 & 1.4 & & 7 \\
\hline 0.01 & 0.01 & 4 & 0.7 & - & 0.8 & 2 \\
\hline 0.01 & 0.01 & 2 & 0.7 & 1.4 & - & 15 \\
\hline 0.01 & 0.01 & 2 & 0.7 & - & 0.8 & 7 \\
\hline 0.01 & 0.01 & 1.5 & 0.7 & 1.4 & - & 27 \\
\hline 0.01 & 0.01 & 1.5 & 0.7 & - & 0.8 & 12 \\
\hline 0.01 & 0.01 & 1.0 & 0.7 & - & 0.8 & 28 \\
\hline
\end{tabular}

Tabla 1. Tamaño de muestra para comparación de Medidas

Error tipo I: Rechazar la hipótesis nula cuando es verdadera.

Error tipo II: Aceptar la hipótesis nula cuando es falsa.

Diferencia: Diferencia hipotética considerada significante. (Diferencia de gramos desbastados entre limas del fabricante $A$ y las de la competencia).

Desviación estándar: Desviación estándar de gramos desbastados por las limas

La tabla 1 sugiere un tamaño de muestra de por lo menos 27 para comparar las limas del fabricante $A$ con las del fabricante $B$, suponiendo una diferencia entre sus rendimientos de 1.5 gramos/1000 ciclos, errores de 1\% y un tamaño de muestra de por lo menos 28 para comparar las limas del fabricante $A$ con el fabricante $C$, suponiendo una diferencia de rendimiento de 1 gramo/1000 ciclos y errores del 1\%. De acuerdo con los resultados estadísticos obtenidos se sugieren 30 limas por cada compañía para que las mediciones sean estadísticamente confiables.

La información tomada se resume en la Figura 2, donde se muestra el peso inicial de la probeta, el final y los gramos de material removido por cada 1000 ciclos de operación para las limas de cada fabricante. Esta información fue tomada como base para los análisis estadísticos. 


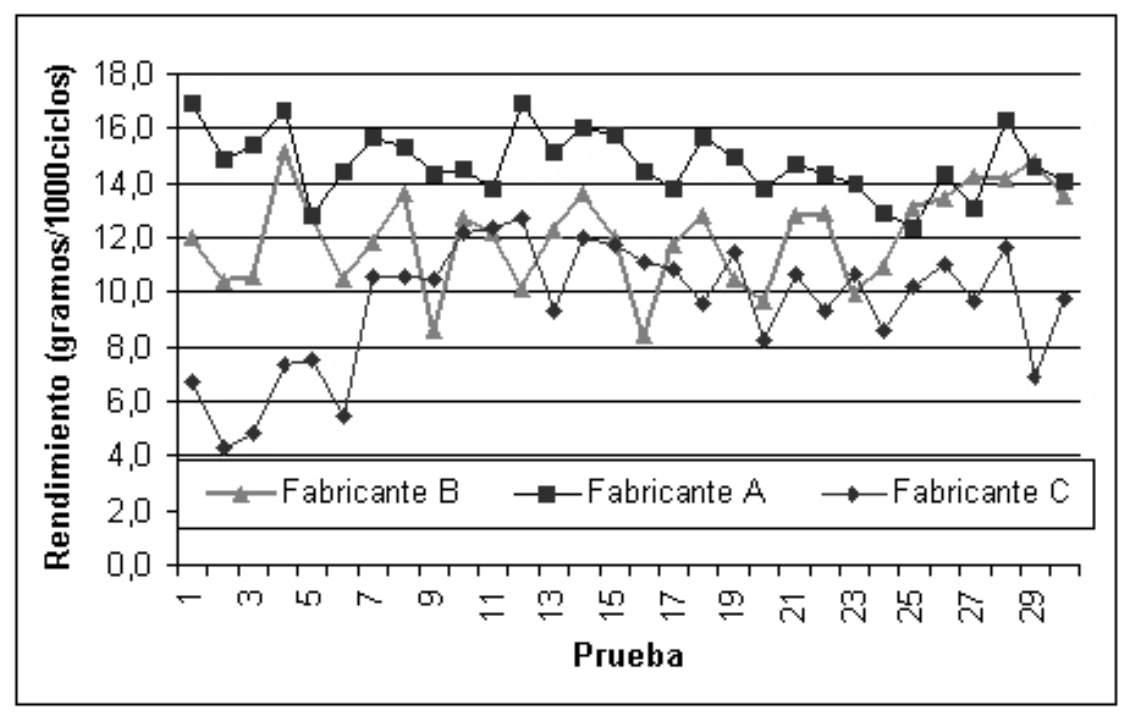

De la Tabla 2 se puede concluir que el rendimiento de las limas del fabricante $B$ está comprendido entre 11.38 y 12.69; las del fabricante $A$ entre 14.29 y 15.17 y las del fabricante C entre 8.74 y 10.44 , todos estos intervalos con una confiabilidad del 95\%. Lo anterior indica que para esta prueba las limas del fabricante A presentan un rendimiento promedio superior del orden del $18.77 \%$ con respecto a las del fabricante $B$ y $53.60 \%$ con las del fabricante $C$.

Figura 2. Rendimientos de Limas

\section{Estimación del rendimiento}

Si $\bar{x}$ y s son la media y la desviación estándar de una muestra aleatoria de una población normal con variancia desconocida $\sigma^{2}$, un intervalo de confianza del (1- $\alpha) 100 \%$ para la media poblacional $\mu$ es:

$$
\bar{x}-t_{\alpha / 2} \frac{s}{\sqrt{n}}<\mu<\bar{x}+t_{\alpha / 2} \frac{s}{\sqrt{n}}
$$

Donde $t_{\alpha / 2}$ es el valor de $t$ con $v=n-1$ grados de libertad ( $n$ : tamaño de la muestra aleatoria, $\alpha$ : nivel de incertidumbre).

Para $n=30$ se obtiene $v=30-1=29$ y usando información sobre valores críticos de la distribución $t$, con un nivel de incertidumbre $\alpha=0.05$ se obtiene $t_{\alpha / 2}=t_{0.025}=2.045$. Así el intervalo de confianza para el rendimiento de la población de limas utilizando la ecuación 1 es:

\begin{tabular}{|c|c|c|c|c|c|}
\hline LIMAS & \multicolumn{5}{|c|}{ IMTERVALO DE COMFIAMZA } \\
\hline Fabricante A & 14.29 & $<$ & $\mu$ & $<$ & 15.17 \\
\hline Fabricante B & 11.38 & $<$ & $\mu$ & $<$ & 12.69 \\
\hline Fabricante C & 8.74 & $<$ & $\mu$ & $<$ & 10.44 \\
\hline
\end{tabular}

Tabla 2. Intervalo de confianza para el rendimiento poblacional de limas $(\alpha=0.05)$

\section{Estimación de la diferencia de rendimientos}

Para cuantificar la superioridad del rendimiento de las limas del fabricante $A$, respecto a las del fabricante $B$ y fabricante $C$, es necesario realizar una estimación de diferencias entre rendimientos, lo cual se logra a partir de un análisis de estimación o de prueba de hipótesis.

Si $\bar{x}_{1}$ y $s_{1}^{2}, \bar{x}_{2}$ y $s_{2}^{2}$, son las medias y variancias de muestras pequeñas independientes de tamaños $n_{1}$ y $n_{2}$ respectivamente, de distribuciones aproximadamente normales con variancias diferentes y desconocidas, un intervalo de confianza aproximado de (1- $\alpha) 100 \%$ para la diferencia de medias poblacionales $\mu_{1}-\mu_{2}$ está dado por:

$\bar{x}_{1}-\bar{x}_{2}-t_{\alpha / 2} \sqrt{\frac{S_{1}^{2}}{n_{1}}+\frac{S_{2}^{2}}{n_{2}}}<\mu_{1}-\mu_{2}<\left(\bar{x}_{1}-\bar{x}_{2}\right)+t_{\alpha / 2} \sqrt{\frac{S_{1}^{2}}{n_{1}}+\frac{S_{2}^{2}}{n_{2}}}$

Donde $t_{a / 2}$ es el valor t con grados de libertad,

$$
\mathcal{V}=\frac{\frac{s_{1}^{2}}{n_{1}}+\frac{s_{2}^{2}}{n_{2}}}{\left[\left(s_{1}^{2} / n_{1}\right) /\left(n_{1}-1\right)\right]+\left[\left(s_{2}^{2} / n_{2}\right)^{2} /\left(n_{2}-1\right)\right]}
$$


Para $n_{1}=n_{2}=30^{1}$, el nivel de incertidumbre $\alpha=0.05$, tomando los valores necesarios de la tabla 2 y usando la ecuación 2 , se obtienen los resultados que se muestran en la tabla 3.
DOEBELIY, Ernest, "Engineering Experimentation, planning, execution, reporting", McGraw Hill, 1995.

MICHELETTI G. F., "Mecanizado por Arranque de Viruta", Editorial Blume, 1980.

\begin{tabular}{|c|c|c|c|c|c|c|c|}
\hline LIMAS & \multicolumn{5}{|c|}{ INTERVALO DE COMFIAMZA } & $\boldsymbol{v}$ & $\boldsymbol{t}_{\alpha / 2}$ \\
\hline Fabricante B & 1.94 & $<$ & $\mu_{1}-\mu_{2}$ & $<$ & 3.45 & 51 & 1.96 \\
\hline Fabricante C & 4.22 & $<$ & $\mu_{1}-\mu_{2}$ & $<$ & 6.06 & 54 & 1.96 \\
\hline
\end{tabular}

WALPOLE E. Ronald, "Probabilidad y

Tabla 3. Intervalo de confianza para la diferencia de rendimientos $(\alpha=0.05)$

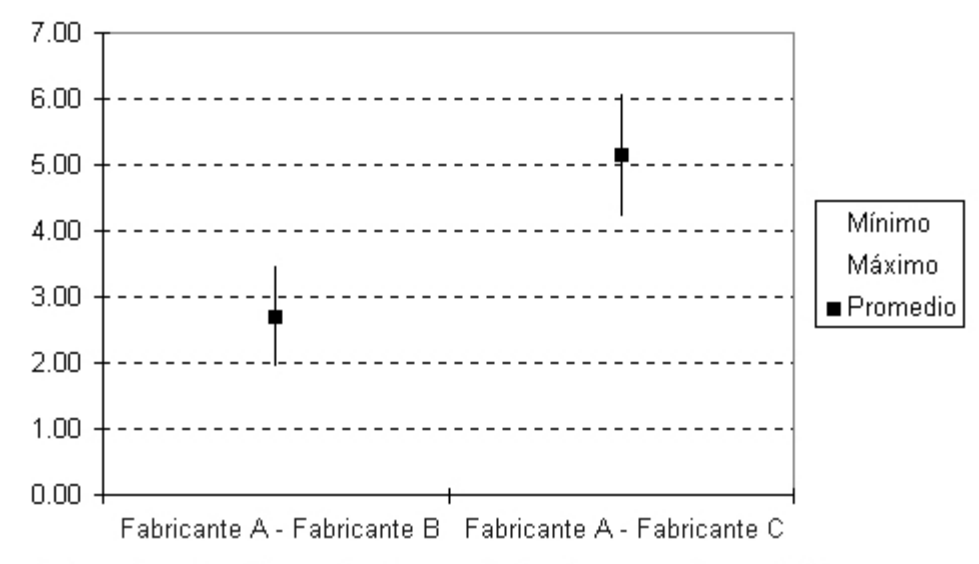

Intervalos de diferencia de rendimientos para limas RT6

Figura 3. Intervalos de diferencia de rendimientos para limas RT6

De la tabla 3 se puede concluir con una confianza del $95 \%$ que el rendimiento de las limas del fabricante $A$ superan a las limas del fabricante $B$ entre 1.94 a 3.45 (gramos removidos/ 1000 ciclos) y a las limas del fabricante $C$ entre 4.22 a 6.06. (ver Figura 3). Es decir que para esta prueba las limas del fabricante $A$ son superiores a las del fabricante $B$, entre un 16.1 y $28.7 \%$ y a las del fabricante $C$, entre un 44.0 y $63.1 \%$.

\section{BIBLIOGRAFÍA}

DÍAZ, Abel, "Diseño Estadístico de Experimentos". Editorial Universidad de Antioquía, 1999.

HAUGEM, Edward, "Probabilistic Mechanical Design", John Wiley \& Sons, Inc, 1980. 